\title{
On the design of an energy-efficient low-latency integrated protocol for distributed mobile sensor networks
}

\author{
A.G. Ruzzelli†, L. Evers, S. Dulman, L.F.W. van Hoesel, P.J.M. Havinga \\ Department of Computer Science, University of Twente \\ The Netherlands \\ $\nmid$ Department of Electronic Engineering, Universita' degli studi di Ferrara \\ Italy \\ \{ruzzellig, eversl, dulman, hoesel, havinga \}@es.utwente.nI
}

\begin{abstract}
Self organizing, wireless sensors networks are an emergent and challenging technology that is attracting large attention in the sensing and monitoring community. Impressive progress has been done in recent years even if we need to assume that an optimal protocol for every kind of sensor network applications can not exist. As a result it is necessary to optimize the protocol for certain scenarios. In many applications for instance latency is a crucial factor in addition to energy consumption. MERLIN performs its best in such WSNs where there is the need to reduce the latency while ensuring that energy consumption is kept to a minimum. By means of that, the low latency characteristic of MERLIN can be used as a trade off to extend node lifetimes. The performance in terms of energy consumption and latency is optimized by acting on the slot length. MERLIN is designed specifically to integrate routing, MAC and localization protocols together. Furthermore it can support data queries which is a typical application for WSNs. The MERLIN protocol eliminates the necessity to have any explicit handshake mechanism among nodes. Furthermore, the reliability is improved using multiple path message propagation in combination with an overhearing mechanism. The protocol divides the network into subsets where nodes are grouped in time zones. As a result MERLIN also shows a good scalability by utilizing an appropriate scheduling mechanism in combination with a contention period.
\end{abstract}

keywords: Medium access control, routing, lo- calization, mobile sensor networks, energy efficient, lightweight protocol, low latency.

\section{Introduction}

WSNs consist of a large number of wireless sensors, we shall refer to as nodes, and a fixed number of gateways or beacons. Communication between a gateway and nodes needs to be bidirectional. A node is a small electronic or electromechanical device with processor and transceiver. A node is able to communicate only within a limited range, hence when a particular sensor is asked to send a packet, it can transmit only to neighboring nodes. As a result, packets hop from node to another till they get to a gateway. Nodes may have attached sensing devices like a magnetometer, light detector, compass, thermistor etc. depending on the requirement of the application. Nodes can be positioned on objects or animals hence we assume they are mobile. WSNs are controlled and monitored by one or more computers (with a user interface)connected to the gateways. Communication is based on request from a user to one or more nodes in the network.

A big effort and interest is being focused on this new topic mainly due to many useful application related to:

- Environmental monitoring for fire prevention (e.g. in national parks, forest etc.)

- distributed monitoring in agricultural plantations;

- distributed monitoring of chemical processes; 
- Animal herd monitoring for instance by putting such cheap sensors on cows;

- Monitoring remote inaccessible areas like volcanoes or extraterrestrial planets. Then the deployment of such network can be done by dropping sensors for example from a plane;

- Medical monitoring. The sensors can be put on a patient bodies and in their houses. In this way a doctor can check his patient conditions (heart beats number, temperature etc.) from an away office.

- Emergency operations like disaster relief operation.

- Indoor monitoring. WSNs can be a valuable alternative to wired networks to minimize the infrastructure installation costs and maintenance.

In most of such applications energy is a scarce resource. Nodes have special low power hardware and batteries may be difficult or impossible to recharge hence saving energy is of paramount importance. Since radio communication uses the majority of power, during transmitting, listening and receiving, minimizing the radio activity is the major target to pursue. WSNs usually are known to have low bandwidth and high latency. However, many applications require a fast response so reducing the latency is another target to pursue. Moreover another characteristic to be mentioned is the limited memory capacity of cheap wireless sensors.

We highlight the major forms of energy wastage :

Idle listening It is one of the main sources of energy wastage mainly due to the fact that nodes listen to the channel even if no packets have been sent.

Collisions When two ore more nodes transmit simultaneously, packets interfere, so they need to be retransmitted as a result.

Overhead To avoid collision and to coordinate nodes, some protocols use control packets. As a. result nodes activity increases.

Overhearing It happens when some nodes can listen to packets that are not destined for them.
To satisfy all these requirements, we have developed MERLIN which is a flexible, lightweight and integrated protocol that combines MAC, routing and localization protocols.

Many articles and publications about WSNs have been written so far (see the related work section), most of them try to make a compromise between energy consumption, latency, scalability and reliability of the network. The MERLIN protocol focuses on latency while ensuring that node lifetime is kept to a maximum. No explicit handshake mechanism is used among nodes. Initially this may be seen to reduce the reliability of transmission. On the contrary reliability is achieved by an implicit handshake mechanisin and by relying on multiple paths of the network that exploit time-zones. As a result while node lifetime increases, there is a decrease of overhead and latency.

MERLIN is the combination of a TDMA and CSMA approaches. The big advantage of this is that while the TDMA approach saves energy and avoids many collisions, the CSMA approach increases the scalability of the protocol. MERLIN exploits the CSMA approach by a contention period at the beginning of each slot where nodes listen to the channel. A detailed explanation is given in section 4.2 .

One of the most important base services that a WSN should provide, is some way to identify the participating nodes, based on their position. In MERLIN, localization is an integrated part of the system, providing an efficient way to obtain the nodes positions. The localization procedure used is based on earlier work by Evers [3], enhanced in a way that allows the network to be able to deal with moving nodes. Further details can be found in Section 3.

\section{Related work}

Extending node lifetime is the main aim of WSN protocols. For this reason protocols like IEEE802.11 [5] or MACAW [1], which uses CSMA with a handshake mechanism, is not suitable. One of the earliest protocols for ad hoc WSNs was PAMAS [11] that proposed a contention based algorithm to save energy. Recent research initiatives in the field of WSNs suggest methods to decrease the energy consumption of nodes. SMAC by Ye [14] 
for example uses a coordinated adaptive sleeping mechanism. The main drawbacks are the latency because of a RTS/CTS mechanism and the increase of energy consumption when some nodes join the network. This is mainly due to the CSMA approach. TRAMA [10] helps to improve of nodes lifetime but introduces a high complex slot assignment algorithm. A very recent protocol is DMAC $[6]$, which has a sort of data gathering tree that reduces the latency. The drawback is that DMAC is suitable only for unidirectional communication flow to a single gateway. Overhearing improvement of SMAC can be found in TMAC [2], even though energy wastage due to RTS/CTS collisions and very high latency are still present. Tiny-DB [7] describes a similar division of the medium to collect data like MERLIN. The author tries to helps to reduce the contention in the MAC protocol by dividing the network area in levels. The thesis refers to the Woo and Culler MAC [12], which examined different configurations of CSMA and RTS/CTS to find a fair bandwidth allocation to all nodes. The authors did not implement any mechanism like multiple path or overhearing mechanisms to increase the reliability and to reduce the overhead. Furthermore, tiny-DB has been designed on top the MAC protocol which may cause latency problems.

MERLIN also compete with EMACS [4] and ESR routing [13] as the communication protocol designed and implemented on the EYES sensor node [9]. The improvement in using MERLIN are the lower latency and lightness. In fact, memory of wireless sensors is small (EYES nodes run with $2 \mathrm{~KB}$ of $\mathrm{RAM}$ ) hence communication protocols should use it as little as possible.

\section{Assumption}

This section gives the details of some assumption that we made to develop the MERLIN protocol.

EYES sensors are equipped with transceiver, processor and memory. Their cost is kept to a minimum hence memory and energy are scarce resources. We assume a single channel transceiver that can operate in three states: transmit, receive and standby. Transmitting consumes more power than receiving and standby lies beneath the power used for receiving roughly by a factor of 1000 . The below transceiver data sheet in Table 1 gives some extra information about the energy consumption.

\begin{tabular}{|c|c|}
\hline Parameter & Value \\
\hline Energy constumption transmitting & $21 \mathrm{~mW}$ \\
Energy consumption receiving & $14.4 \mathrm{~mW}$ \\
Energy consumption standby & $15 \mu \mathrm{W}$ \\
Switch time transmit/receive & $518 \mu \mathrm{s}$ \\
Switch time receive/transmit & $12 \mu \mathrm{s}$ \\
Switch time standby/receive & $518 \mu \mathrm{s}$ \\
Switch time standby/transmit & $16 \mu \mathrm{s}$ \\
\hline
\end{tabular}

Table 1: EYES prototype data sheet

In order to integrate the $\mathrm{MAC}$, routing and localization protocols, MERLIN is designed to optimize the following data traffic patterns:

Subnet flooding by gateway Gateway packets are forwarded to all nodes in the subnet;

Local broadcast by node Nodes send packets to all of the direct neighbors. No forwarding is performed;

To gateway Transmission by node Nodes closer to the gateway forward the packet until it reaches the gateway.

MERLIN could be also adapted to different types of data queries that may be injected into the network. The above data traffic are performed by means of the transmission mechanism described in Section 4.2. This data traffic is quite typical for many WSNs applications and algorithms.

As previously mentioned, MERLIN is designed to provide a localization algorithm integrated. The procedure works efficiently doing "local broadcast by node" which is also used to update the synchronization and the time-zone of nodes. As a starting point, we assume gateways know their positions, either manually configured, read from a GPS receiver or something alike. Upon arrival of a radio packet, the received signal strength is measured, and used to calculate an estimation of the distance between the nodes. These distances, along with accuracy of the distance estimation, are communicated across the network. When the number of measurements is enough then a node can calculate its position and broadcast it. Neighboring nodes will use this position, along with the measured distance towards it, to calculate their position and broadcast it. Nodes will keep on forwarding measured distances, until 


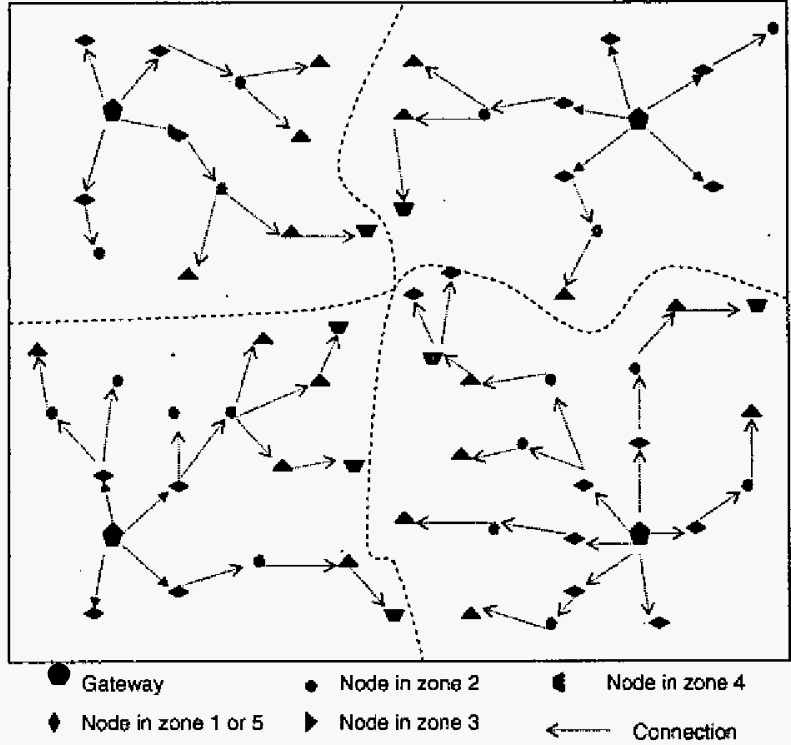

Figure 1: The above picture shows the division of the network after the network flooding by gateways. Every node sets its zone and forward the packet

all nodes have calculated their own position. When a distance between nodes seems to have changed significantly, the surrounding nodes will recalculate their distances, and rebroadcast it.

\section{Design Of The MERLIN Protocol}

\subsection{Overview}

This section gives an overview of MERLIN and explains the reason why certain choices have been made. Further details will be given in the following sections.

First, the main idea behind MERLIN is the natural division of the network in subnet which are created when one or more gateways start transmitting, as shown in Figure 1. In this way nodes set their time-zone number as the number of hops to get to the closest gateway. Once a node learns which time-zone it belongs to, it would immediately know which schedule to use to communicate with neighboring nodes and can thus join the network. Carrier sensing allows nodes to avoid colli- sions. Moreover an additional simple collision detection improves the reliability. The gateway provides time synchronization among all nodes in the network which is essential element of the time division mechanism.

Second, we describe the routing features of MER LIN to perform a reliable and efficient communication in terms of energy consumption and latency. The communication to the user is done with a set of powerful nodes: The gateways. User requests are flooded into the network to all nodes. Node replies are forwarded to gateways over several hops. For this reason, nodes decide to forward the packet based on their time zones (i.e. number of hops) needed to get the gateway:

Third, we outline the localization procedure based on triangulation and distance between pairs of nodes. Just few nodes positions are needed for the MERLIN localization procedure; the rest is calculated based on broadcast communication. As a result, localization procedure relies on fixed gateways as their positions are known. They provide access to the user, shared time base and localization anchors.

Next, as a result of the division of the network in time-zones then in subnets, we define:

Time-zone features

- A time-zone is equal to the number of hops to the closest gateway hence, nodes in the same time-zone have the same hop count number;

- Time-zones are set-up with a subnet flooding by the gateway and updated by regular communication;

- Nodes within the same time zone own the same slot;

- Nodes belong to one gateway only;

- Nodes store the number of hops to the closest gateway.

\subsection{MERLIN MAC Detail}

This section describes the basic mechanisms used by MERLIN to avoid collisions. In particular, it explains the usage of the contention period and the collision reporting period at the beginning and at the end of each slot. 
After the initialization procedure, the network is divided into subnets. Only one gateway is present in each subnet. Nodes are organized in time-zones.

The node time-zone is set-up when a "subnet flooding by gateway" takes place and is updated doing regular communication and by periodical local broadcasts by the nodes. During a transmission, a packet hops from one node to another until it gets to the destination.

\subsubsection{MERLIN TDMA/CSMA based ap- proach}

Time is divided into slots, with fixed starting times and length (in our implementation slot length $=$ $0.25 \mathrm{~s}$ ). MERLIN does not perform RTS/CTS to save time and energy. Nodes can transmit in one slot by contending for the channel with neighbors. Slot contention is performed at the beginning of each slot where a contention period (CP)is present. This period is much shorter than the entire slot and it is used to avoid collision between neighboring nodes. All wireless nodes must be well synchronized between themselves and with the gateways. For this reason MERLIN includes a synchronization mechanism described in Section 4.5.

The steps taken by a node that wants to transmit are :

1. Pick a random time $T$ during the contention period $\mathrm{CP}^{1}$.

2. Listen to the channel from the beginning of the contention period.

3. If nothing is heard until the time $T$ then the node starts sending its packet.

4. If the node listens to a neighbor, which start sending, it means another transmission is on, and the node keeps on listening to the msgindex. The node will turn off the radio afterwards. The msg-index is an index containing the ID of messages included in the packet as described in Section 4.8. In this case the contention procedure will be repeated in the next designated slot.

If a node is scheduled to receive, it wakes up to listen to the CP. If no msg is heard by the end of

\footnotetext{
${ }^{1}$ Contention period
}
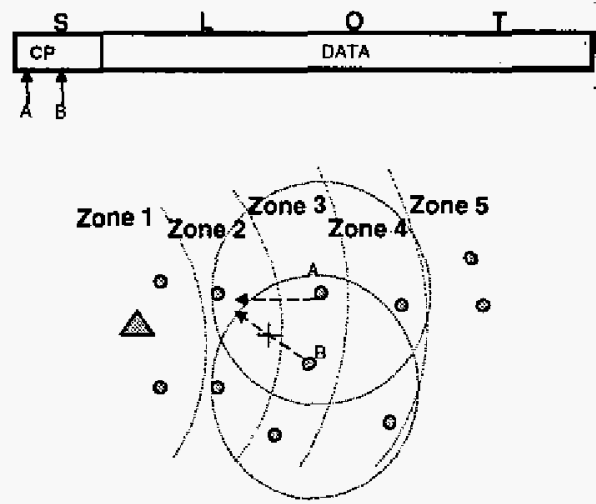

Figure 2: Node A and B want to transmit. They pick up a random time in the contention period. $A$ and B start listening to the channel from the beginning of the contention period. Only if the channel is free at the time they picked up, the node can start sending its packet. In the above example A will transmit while $B$ has to wait until its next scheduled slot.

the CP then the node turns off the radio until the next slot scheduled. Further explanation is given in Figure 2.

\subsubsection{Collision reporting}

In several situation collisions can still occur because of hidden terminal problems and for not listening while sending. In such cases transmitting nodes do not know that a collision has occurred. For this reason, every slot has a collision reporting period (i.e. $\mathrm{CR}^{2}$ ) positioned at the end as shown in Figure 3. A node that hears the collision sends a $C R$ at the end of the slots in order to notify all senders that a collision has taken place. All senders need to listen during that period. In case of collision, the packet will be rescheduled after a random exponential backoff procedure. The collision report consists of a short burst message.

\subsection{MERLIN Routing Detail}

MERLIN includes an implicit routing mechanism. In fact, the division of the network in time-zones would allow a node to be aware that its transmitted message is propagating either to or away from a gateway. Such a mechanism is due to the fact that:

\footnotetext{
${ }^{2}$ Collision report
} 


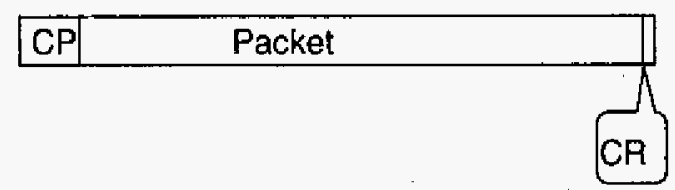

Figure 3: The above figure shows the position of the collision report period that receiving nodes use to broadcast a burst message in case of collision

- Nodes store their time-zone (hop count number) updating it during regular transmission;

- Nodes include their hop count number in every packet they send so that receiving nodes can update their time-zone;

- Node hop count is calculated as:

Node Hop Count $=$ min (hop count of neighbors) +1 ;

- Gateway hop count is set as zero;

- Packets can be forwarded in two directions only :

Toward the gateway or away from it;

- For "To gateway transmission", nodes forward the packet only if sent from a node with higher hop count than its own

- For "Subnet flooding by gateway", nodes forward the packet only if it is sent from a node with lower hop count than its own.

\subsection{MERLIN Scheduling}

The purpose of MERLIN scheduling is the timezone slot allocation. In fact, it has been already said that nodes in the same time-zone use the same slots to transmit. The schedule table is performed such that it results in fast transmission (i.e low latency)and it reduces collisions.

Nodes send only in one slot per frame according to the hop count. By definition all neighbors of a node are within one hop count difference. The schedule table is designed to prevent nodes with hop count difference of two (i.e. within 2 time-zone) to transmit simultaneously. The schedule table is built in a way that allows messages to be forwarded one slot after they received toward the gateway or away from it. Localization is performed by local . broadcast messages to all neighbors.

Two schedule tables are presented as an example. The slot allocation described is for 4 timezones. Slots for further time-zones can be allocated appending the same table. Routing is optimized in the direction toward the gateway ( / ) or away from it $(\backslash)$. This can be depicted like a V-shape in which the up and downlink traffic is sequential or like a X-shape in which up and downlink traffic is combined.

Table 2 shows the V-table that performs better in terms of number of collisions and energy consumption but worse than $\mathrm{X}$-table in terms of latency and throughput. We stress the fact that latency is still very low than other WSN protocols.

The table 3 called X-table performs better in terms of throughput and latency. Few collisions are reported at the intersection point where adjacent nodes try to send one opposite the other. In fact, most of them are prevented by the contention period that allows only one of those to transmit. In accordance with the requirements of the application, many other schedule tables can be built by allowing more transmission to the gateway or away from it. Furthermore, sleeping time of nodes can be decided by adjusting the slot length. These two adjustments increase the flexibility of MERLIN with respect to the application.

\begin{tabular}{|c|c|c|c|c|c|c|c|c|}
\hline & siot 1 & slot2 & slot3 & slot 4 & stot & slot2 & slot:3 & silot \\
\hline $\begin{array}{l}\text { Zone } \\
\text { n }\end{array}$ & $T \times \downarrow$ & $\mathrm{Rx}$ & & $\mathrm{Rx}$ & $\mathrm{Rx}$ & & $\mathrm{Rx}$ & $\operatorname{Tx} \uparrow$ \\
\hline $\begin{array}{l}\text { Zone } \\
\mathrm{n}+1\end{array}$ & $\mathrm{Rx}$ & $\mathrm{Tx}$ & $\mathrm{Rx}$ & & & $\mathrm{Rx}$ & $\mathrm{Tx}$ & $\mathrm{Rx}$ \\
\hline $\begin{array}{l}\text { Zone } \\
n+2\end{array}$ & & $\mathrm{Rx}$ & $\mathrm{T} \times \|$ & $\mathrm{Rx}$ & $\mathrm{Rx}$ & $\overline{T x} \uparrow$ & $\mathrm{Rx}$ & \\
\hline $\begin{array}{l}\text { Zone } \\
n+3\end{array}$ & $\mathrm{Rx}$ & & $\mathrm{Rx}$ & $\mathrm{Tx}$ & $\mathrm{Tx}$ & $\mathrm{Rx}$ & & $\mathrm{Rx}$ \\
\hline
\end{tabular}

Table 2: fast performing V-scheduling

\subsection{Timing and Synchronization}

All nodes in the network have to agree to the starting time of slots, in order to correctly receive each others packets. This starting moment of the slots has to be communicated among nodes. Several methods of time synchronization have been devel- 


\begin{tabular}{|l|l|l|l|l|}
\hline & slot1 & slot2 & slot3 & slot4 \\
\hline $\begin{array}{l}\text { Zone } \\
\mathbf{n}\end{array}$ & $\mathrm{Tx} \downarrow$ & & $\mathrm{Rx}$ & $\mathrm{Tx} \uparrow$ ox $\mathrm{Ax}$ \\
\hline $\begin{array}{l}\text { Zone } \\
\mathbf{n}+1\end{array}$ & $\mathrm{Rx}$ & $\mathrm{Tx} \downarrow$ or $\mathrm{Rx}$ & $\mathrm{Tx} \uparrow$ & \\
\hline $\begin{array}{l}\text { Zone } \\
\mathbf{n}+2\end{array}$ & $\mathrm{Rx}$ & $\mathrm{Tx} \uparrow$ or $\mathrm{Rx}$ & $\mathrm{Tx} \downarrow$ & \\
\hline $\begin{array}{l}\text { Zone } \\
\mathbf{n}+3\end{array}$ & $\mathrm{Tx} \uparrow$ & & $\mathrm{Rx}$ & $\mathrm{Tx} \downarrow$ ox $\mathrm{Rx}$ \\
\hline
\end{tabular}

Table 3: fast performing $\mathrm{X}$-scheduling

oped already, all with different purpose and applicability to the MERLIN system. Among those, the NTP protocol [8] is the most widespread, providing the standard of time synchronization across the internet. The hardware involved in the timing aspects of the EYES-nodes is low-cost low-energy. As a consequence, the frequency with which the timers will run differs slightly from node to node, as well as over time, due to temperature differences etc. Besides synchronizing the starting moment (offset) of the slots among nodes, the frequency skew of each node's individual clock needs to be compensated for continuously. Both of these issues are solved by including the time of transmission on every radio transmission. All receiver nodes can then estimate the start of the slot according to the sender, and synchronize their clock offset. Furthermore, the clock skew can be estimated by comparing over time the observed difference between a senders clock and the node's own clock. The gateways are used to provide the best time synchronization, as we have assumed them to know the "perfect time". The hop count 1 nodes can then update their clock offset and skew, by comparing it to the observed time from messages received from the gateway. In general, nodes update their clock synchronization from the nodes nearer to the gateways. The time zones form a hierarchy, providing better synchronization for zones with small hop counts, much alike the stratums used in NTP. At initialization, a node cannot participate in sending into the network, until it has been properly synchronized to the start of the slots. In MERLIN, the first message received by a node from another node already synchronized, can provide the clock offset, after which the node can participate in the network, and forward the message just received. Subsequent

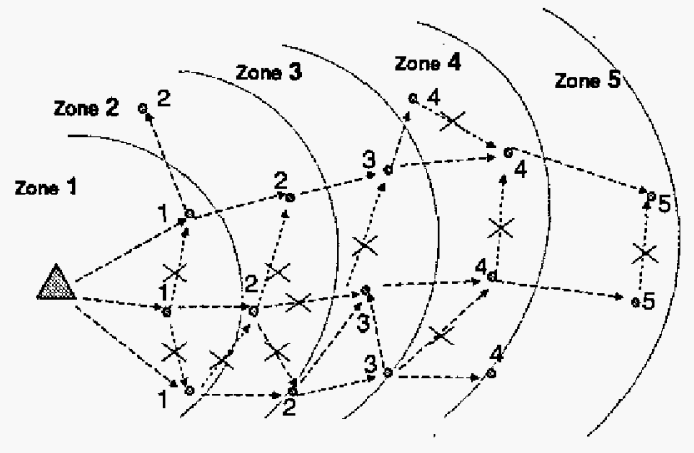

Figure 4: The above picture shows how a packet of the gateway floods the network. Every node receiving the packet set its time-zone, update the hop count of the packet and forward it. Some communication fail because nodes can be in the same time-zone or because of the contention period.

messages will allow the node to estimate its clock skew as well.

\subsection{Network initialization and setup}

MERLIN does not need a proper initialization procedure. At the beginning of each session, nodes are already in the area waiting for any kind of signal from gateways. At that moment they are isolated. The only important thing to notice is the fact that fixed gateways must be tightly synchronized. This means that gateways know the "perfect time". In order to set the network one or more gateways flood the network with a message: Nodes will set their hop count number or the time-zone. Nodes update the message with their hop count number and forward it.

The message sent by gateway includes synchronization information (i.e. sync-info) with respect to it. Sync-info are included in every message transmitted also in regular communication. Hence a new node that wants to join the network has to listen to the channel for any message, obtain the sync-info and process its time zone from it.

An new gateway that wants to join the network, firstly has to listen to the channel and realize its time zone as a node. Secondly, it announce its presence sending its message (with sync-info included). Neighboring nodes realize the presence of the new gateway, process the new sync-info and forward the message. Nodes will compare the distance with re- 
spect to the old and new gateway by looking at the two time-zones. Nodes will change their time-zone, hence their schedule table, at the beginning of the successive frame only if the transmission have been successful and in case the new gateway is closer.

This procedure allows the division of the network in "subnet" with a gateway in each of those. In each subnet nodes are organized in time zones.

Since sync-info are included in every message, node positions are updated automatically with normal communication and doing periodical local broadcast by nodes. Such a procedure is also used for the localization as already described in section 3.

\subsection{MERLIN Implicit multiple path}

In order to describe the implicit multiple path generation, we make a distinction between a message and a packet.

- A message is generated by one node and it is identified by a combination of a source ID and message ID.

- Nodes have the option of concatenating several messages. and then transmit them as a single packet. In fact, if a node has more than one message to send, it will send them as a packet in the same slot thus reducing transmission overhead, which in turns results in energy savings. To optimize the protocol, packets contain a msgs-index that is positioned at the beginning of the slot as shown in Figure 5 .

Forwarding "transmission to gateway" by nodes results in copies of same message. Nodes can detect copies of arriving msgs by combination of Source ID and message ID contained in the msg. As nodes are disjoined, messages arriving at gateway follow multiple paths. In this way packets arrive at the gateway following different paths. The advantage of multiple path propagation is the greater reliability while the disadvantage is the increase of overhead. To reduce it we have implemented an overhearing method discussed in the following section.

\subsection{Overhearing}

Nodes not getting the slot keep on listening to the beginning of others transmitting packet. As we de-

\begin{tabular}{|c|c|c|c|c|}
\hline$p$ & $a$ & c & $k$ & $\theta$ \\
\hline Msg-index & & & & \\
\hline
\end{tabular}

Figure 5: The above figure shows the data concatenation packet where it is shown the position of the msgindex at the beginning of the packet

scribed in section 4.2 .1 , nodes not getting the channel receive the packet of the neighbor and turn off the radio. Indeed, nodes need only to get the msgsindex of sent packet. In this way a node realizes whether the packet contains useful msg (e.g. for localization). If the sent packet contains one or more same msgs that the node want to send in its packet, those msgs can be discarded. The msg-index is an index containing the ID of included msgs that is sent at the beginning of the slot as shown in figure 5.

The described MERLIN Overhearing method allows to reduce the overhead in transmission only with a little increase of node activity and complexity.

\section{Implementation and future work}

MERLIN protocol has been designed and is currently implemented using OmNet+t.

Initial results show some collisions on the border of different subnets and around gateways. For these cases, the collision reporting procedure allow the recovery of collided packets. In general, MERLIN seems to follow our expectation.

Some elaborate results in terms of energy consumption, latency and reliability are expected in the nearest future.

The protocol is currently being implemented on a WSNs testbed which contains a small processor (MSP430) with a radio transceiver.

In our future work we plan to work in the following areas :

- A comparison of MERLIN with other recent WSN protocol in terms of energy consumption, latency and reliability;

- Investigate the usage of two different frequencies for subnet flooding and to gateway transmission; 
- Optimize the overhearing mechanism;

- An improved routing protocol that forward the packet looking at the energy consumption of the neighborhood.

- Provide different kind of data queries to inject into the network.

\subsection{Conclusion}

In this paper we have proposed MERLIN as an integrated protocol for WSNs which includes MAC, routing and localization. The division of the network in time-zones by means of a appropriate schedule table reduces considerably the latency without affect the energy consumption. Idle listening is reduced using the TDMA approach, furthermore the CSMA method reduce the number of collisions, put nodes into sleep immediately if no packets has to be forwarded and increase the scalability. In fact, new nodes wishing to join the network only need to listen to the channel and realize their time-zone; no initialization message or procedure is needed. MERLIN is optimized by means of a multiple path mechanism that in collaboration with a overhearing method increase the reliability as well as reduce the overhead in transmission.

\section{Acknowledgments}

This work has been partially supported by the EU as part of the European EYES project (IST-200134734, http://eyes.eu.org). We would like to acknowledge the support of the University of Ferrara through the Leonardo program for the mobility of international students. The authors want to thank Gabriele Lenzini every time present for useful discussion and Suptiyo Chatterjea who has corrected the English grammatical errors thoroughly and with patience.

\section{References}

[1] V. Bharghavan, A. Demers, S. Shenker, and L. Zhang, Macaw: A medium access control for wireless lans, ACM SIGCOMM conference (London UK), September 1994, pp. 212-225.
[2] Tijs Van Dam and Koen Langendoen, $A n$ adaptive energy efficient mac protocol for wireless sensor networks, ACM Sensys (2003).

[3] Leon Evers, Stefan Dulman, and Paul JM Havinga, $A$ distributed precision based localization algorithm for ad-hoc networks, Pervasive2004 (2004), 269-286.

[4] Van Hoesel, Chatterjea, and Havinga, An energy efficient medium access protocol for wireless sensor networks, proRISC 2003 (2003).

[5] IEEE, Wireless lan medium access control and physical layer specification, ANSI/IEEE Standard 802.11 (1999).

[6] G. Lu, B. Krishnamachari, Cauligi, and $\mathrm{S}$. Raghavendra, An adoptive energy-efficient and low-latency mac for data gathering in sensor networks, International workshop on Alghoritms for Wireless, Mobile, ad Hoc Sensor Networks (WMAN 04) (2004).

[7] Samuel Ross Madden, The design and evaluation of a query processing architecture for sensor networks, Ph.D. thesis, University of California, Berkeley, 2003.

[8] Mills, Internet time synchronization. the network time protocolin global states and time in distributed system, IEEE Computer society press, 1994.

9] EYES Project, State of the art, European Union, http//:eyes.eu.org, 2002-2005.

[10] Rajendran, Obrazka, and Garcia-LunaAceves, Energy-efficient, collision-free medium access control for wireless sensor netwoks, Conference on Embedded Networked Sensor System (2003), 181-192.

[11] Singh, Raghavendra, and Pamas, Power aware multi access protocol with signalling for ad hoc networks, Journal (1999).

[12] A. Woo and D. Culler, A transmission control scheme for media access in sensor networks, Mobicom Rome (2001), 221-235.

[13] J. Wu, S. Dulman, and P.J.M. Havinga, $A n$ energy-efficient multipath routing algorithm for wireless sensor networks, Supplement of 
The Sixth International Symposium on Autonomous Decentralized systems, Pisa, Italy (2003).

[14] Wei Ye, John Heidemann, and Deborah Estrin, Medium access control with coordinated adaptive sleeping for wireless sensor networks, Twenty-First AnnualJoint conference of the IEEE Computer and Communication Societies (INFOCOM) 3 (2002), 1567-1576. 